\title{
Erratum to: Nuclear Proteome Analysis of Monkey Embryonic Stem Cells During Differentiation
}

\author{
Davood Nasrabadi • Mehran Rezaei Larijani • \\ Ali Fathi • Hamid Gourabi • Ahmad V. Dizaj • \\ Hossein Baharvand • Ghasem Hosseini Salekdeh
}

Published online: 27 February 2010

(C) Springer Science+Business Media, LLC 2010

\section{Erratum to: Stem Cell Rev and Rep}

DOI 10.1007/s12015-009-9109-6

In the original publication, Dr. Ghasem Hosseini Salekdeh's affiliation was incorrectly given as Karaj, Iran. The correct city is Tehran, Iran.

The online version of the original article can be found at http://dx.doi. org/10.1007/s12015-009-9109-6.

D. Nasrabadi · A. Fathi · G. H. Salekdeh $(\bowtie)$

Department of Molecular Systems Biology,

Royan Institute for Stem Cell Biology and Technology, ACECR,

P.O. Box: 19395-4644, Tehran, Iran

e-mail: Salekdeh@RoyanInstitute.org

M. R. Larijani $\cdot H$. Baharvand $(\square)$

Department of Stem Cells and Developmental Biology,

Royan Institute for Stem Cell Biology and Technology, ACECR,

P.O. Box: 19395-4644, Tehran, Iran

e-mail: Baharvand@RoyanInstitute.org

H. Gourabi

Department of Genetics,

Royan Institute for Reproductive Biomedicine, ACECR,

Tehran, Iran

A. V. Dizaj

Department of Reproductive Imaging,

Royan Institute for Reproductive Biomedicine, ACECR,

Tehran, Iran

H. Baharvand

Department of Developmental Biology,

University of Science and Culture, ACECR,

Tehran, Iran

G. H. Salekdeh

Department of Systems Biology,

Agricultural Biotechnology Research Institute of Iran,

Tehran, Iran 